\title{
Characterization of a Crossed Dipole Resonator Array using a Pulsed Free Space Two-Port Photonic VNA
}

\author{
Fahd R. Faridi and Sascha Preu \\ Technical University of Darmstadt, Darmstadt, Germany
}

\begin{abstract}
We demonstrate a Photonic Vector Network Analyzer (PVNA) capable of measuring a complete set of scattering parameters within the frequency range of $0.2-2 \mathrm{THz}$. The PVNA shows superiority in terms of bandwidth compared to electrical VNAs and is also free from the errors introduced by standing waves, metal waveguides, misaligned flanges, and features less stringent requirements on rigorous and timeconsuming calibration routines. As a proof of principle, we characterize a crossed dipole resonator array using the free space two-port PVNA.
\end{abstract}

(C)2021 IEEE. Published version:

https://ieeexplore.ieee.org/abstract/document/9567495

\section{INTRODUCTION}

$\mathrm{R}$ ECENT advances in terahertz $(\mathrm{THz})$ generation and detection have resulted in growing interest and numerous applications of $\mathrm{THz}$ radiation $(100 \mathrm{GHz}-10 \mathrm{THz})$ in crossdisciplinary fields, leading to a substantial demand for $\mathrm{THz}$ devices and components. Developing these devices and components requires efficient and affordable characterization tools for benchmarking. Most characterization tools are frequency-scaled microwave systems that face challenges in terms of tunability and frequency coverage in the $\mathrm{THz}$ domain. Vector Network Analyzers (VNA) are examples of such kinds of characterization tools that are well established in the microwave range and are now also used in the $\mathrm{THz}$ domain. With current advances in frequency extenders, they can now operate up to $1.5 \mathrm{THz}$ [1]. However, these frequency extender systems have a comparatively small bandwidth of the order of only $50 \%$ of the center frequency due to cutoff towards the lower frequencies and onset of multimodal behavior at the higher frequencies. As a result, a broadband analysis of $0.2-$ $1.5 \mathrm{THz}$ requires five or more extenders, which means the exchange, alignment and calibration process must be repeated at least five times. Moreover, the cost of the system (extender and probes) also increases almost exponentially with frequency. Commercial VNAs also encounter difficulties and errors in parameter extraction due to standing waves, aperture size and alignment of hollow metal waveguides [2], misalignment of waveguide flanges [3] etc.

This report demonstrates that PVNAs are alternative characterization tools in the $\mathrm{THz}$ range at the example of simultaneously obtaining all four S-parameters of a cross dipole resonator (CDR) array. The PVNA uses photoconductors under pulsed operation to generate and detect $\mathrm{THz}$ radiation. It utilizes the basic principle of commercially available $\mathrm{THz}$ TimeDomain Spectroscopy (TDS) systems, which can measure transmission or reflection coefficients analogous to $S_{21}$ and $S_{11}$ parameters, respectively.

\section{EXPERIMENTAL SETUP AND CALIBRATION OF PVNA}

The PVNA contains two ErAs:In(Al)GaAs based photoconductive slotline antennas that generate the $\mathrm{THz}$ pulses and two ErAs:InGaAs based photoconductive H-dipole antennas for detection. Details about the performance of the photoconductive devices are discussed in [4]. A modified Menlo-C-fiber system (center frequency $=1560 \mathrm{~nm}$, pulse duration $=90 \mathrm{fs}$, repetition rate $=100 \mathrm{MHz}$ ) containing two fiber ports and an additional, phase-locked free-space port drive the receivers and transmitters, respectively. The free-space port path incorporates a delay stage for scanning the time delay followed by a beam splitter that splits the signal into two. The $\mathrm{THz}$ path contains two wire grid polarizers on each side of the device under test (DUT) that act as frequency-independent $3 \mathrm{~dB}$ couplers to guide the reflected or transmitted $\mathrm{THz}$ beam towards the receiver. Figure 1 illustrates a simple schematic of the PVNA setup. A more detailed description of the system, as

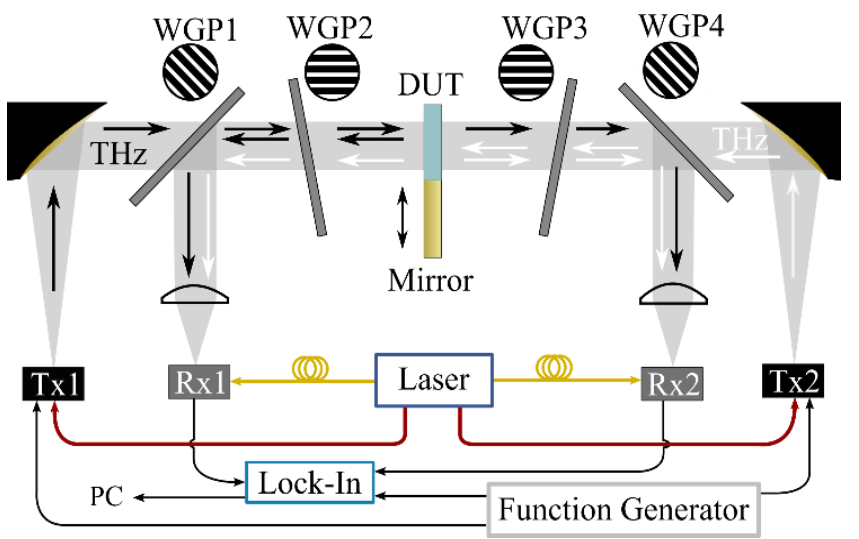

Fig. 1. Experimental setup of a pulsed free space two-port PVNA

well as its attributes, can be found in [5]

For the case of Tx1, the emitted signal that is vertically polarized is first converted to a $45^{\circ}$ linearly polarized signal by WGP1 ( $45^{\circ}$ orientation and $45^{\circ}$ projection on the optical axis) and then again to vertical polarization by WGP2. For each polarization change, the signal is attenuated by at least $3 \mathrm{~dB}$. Now, the vertically polarized signal interacts with the DUT and some part of it gets transmitted through the DUT and some part gets reflected back. The reflected part goes through WGP2 without change and gets deflected at a $90^{\circ}$ angle by WGP1 before finally reaching $\mathrm{Rx} 1$ for detection. The transmitted part and the signal originating from $\mathrm{T} \times 2$ also follow a similar path. Both receivers detect two signals, each from two different transmitters - one is transmitted through and the other is reflected from the DUT, which are distinguished by biasing the transmitters with different modulation frequencies and then demodulating at those frequencies using the lock-in technique. 
The calibration process of the VNA eradicates the systematic error of the measurement such as directivity and cross-talk related to signal leakage, source and load impedance mismatch due to the signal being reflected, frequency response error caused by reflection and transmission tracking with the test receivers etc. Several calibration techniques are widely used for VNAs, such as 'Short-Open-Load-Through' (SOLT) [6], 'Through-Reflect-Line' (TRL) [7], 'Line-Reflect-Line' (LRL) [8] etc. Similar to a commercial VNA, the PVNA calibration procedure removes systematic errors. However, this process is more straightforward for a PVNA. This is mainly due to the fact that the propagation of the signal takes place in air (wave impedance $=376.7 \Omega$ ), whereas for VNA, the signal goes through waveguides that are connected to the DUT. For the PVNA, the calibration procedure using a 'Through' or 'Line' and a 'Reflect' standard is performed. For 'Through' or 'Line', a measurement of the empty setup and for 'Reflect', a measurement with a metal mirror in the position of the DUT is recorded. Conveniently, these signals are simultaneously recorded during the reference measurement, so additional measurements are not required. Mueh et al. [9] have described in detail a similar calibration technique for the case of a 1.5 port setup.

\section{RESULTS}

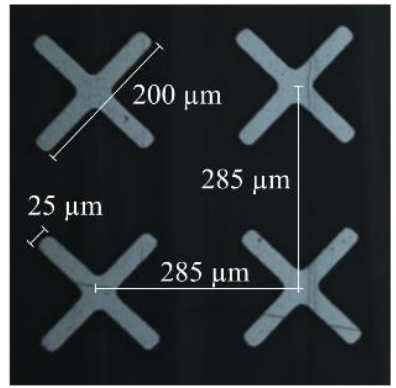

Fig. 2. Section of a CDR array with design parameters.

To verify the system performance, we characterize a CDR array as shown in Figure 2. The array is patterned with $50 \mathrm{~nm}$ thick Aluminium on a $95 \mu \mathrm{m}$ thick HDPE substrate. The Aluminium crosses are separated by a distance of $285 \mu \mathrm{m}$ in both directions with a length and width of $200 \mu \mathrm{m}$ and $25 \mu \mathrm{m}$, respectively. At first, the reference measurements are recorded and then the CDR array is placed in the collimated THz beam and the transmitted and reflected signals are measured. From these measurements, all four S-parameters are calculated. Fig. 3 depicts the measured magnitude and phase of the Sparameters (red) and the simulation (CST Microwave Studio) results (grey). The phase data displayed here are rectified of errors arising from displacement between the position of mirror and DUT (for reflection) and path length difference of measurement and simulation (for transmission). The primary design resonance at around $500 \mathrm{GHz}$ is well reproduced. The resonance peaks show a slight shift and reduced amplitude. The limited spectral resolution of the pulsed PVNA $(7.14 \mathrm{GHz})$ is responsible for the reduced amplitude. The other deviations, however, are due to geometrical imperfections in the produced array and consideration of lossless material in the simulation, proving the necessity of experimental validation. Above 1.5
$\mathrm{THz}$, a relatively large number of waterlines and decreased DNR make it challenging to ascertain the resonance peaks. Still, the measured data show excellent agreement with the simulated results.

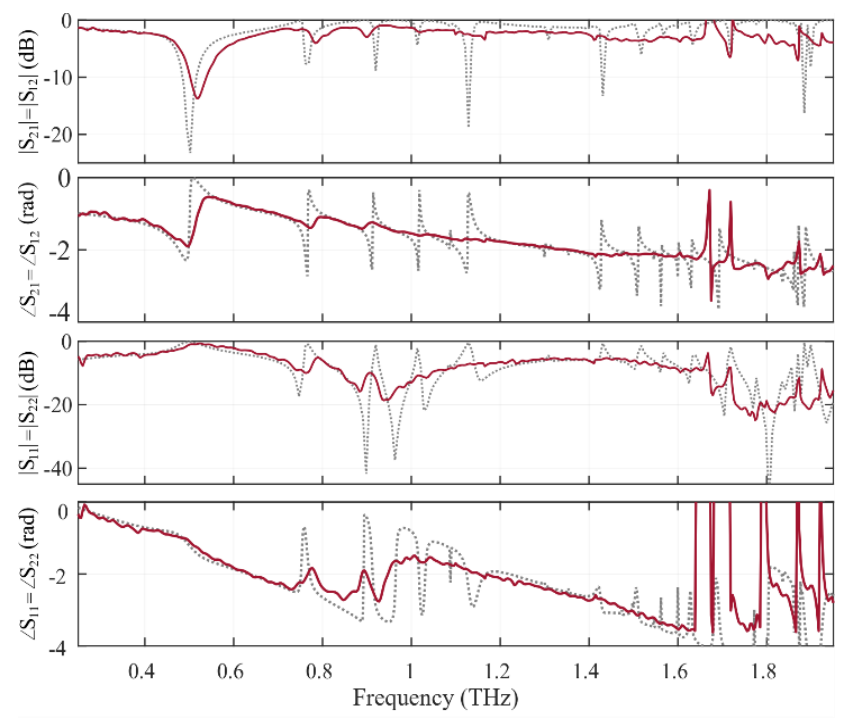

Fig. 3. Measured (red) and simulated (grey) $\mathrm{S}$ parameters (magnitude and phase) of a CDR array. The array is reciprocal, so $S_{21}=S_{12}$ and $S_{11}=S_{22}$.

\section{CONCLUSION}

We demonstrated the high performance of a free space PVNA by measuring the complete set of S-parameters of a CDR array within the $0.2-2 \mathrm{THz}$ range. Huge bandwidth and decent DNR compared with electronic VNA makes the PVNA a better characterization device for broadband investigation.

We acknowledge the European research council for funding the ERC StG 'Pho-T-Lyze', Grant no. 713780 and the Deutsche Forschungsgemeinschaft for funding project PR1413/3-2.

\section{REFERENCES}

[1]. Virginia Diodes, "Vector Network Analyzer Extension Modules (VNAX)." [Online]. Available: https://www.vadiodes.com/en/products/vector-networkanalyzer-extension-modules. [Accessed: 29-Jun-2021].

[2]. N. M. Ridler, S. Johny, M. J. Salter, X. Shang, W. Sun, and A. Wilson, "Establishing waveguide lines as primary standards for scattering parameter measurements at submillimetre wavelengths," Metrologia, vol. 58, no. 1, p. 15015, Jan. 2021

[3]. H. Li, A. Arsenovic, J. L. Hesler, A. R. Kerr, and R. M. Weikle, "Repeatability and Mismatch of Waveguide Flanges in the $500-750 \mathrm{GHz}$ Band," IEEE Trans. Terahertz Sci. Technol., vol. 4, no. 1, pp. 39-48, Jan. 2014. [4]. U. Nandi, J. C. Norman, A. C. Gossard, H. Lu, and S. Preu, "1550-nm Driven ErAs:In(Al)GaAs Photoconductor-Based Terahertz Time Domain System with $6.5 \mathrm{THz}$ Bandwidth," J. Infrared, Millimeter, Terahertz Waves, vol. 39, no. 4, pp. 340-348, Apr. 2018.

[5]. F. R. Faridi and S. Preu, "Pulsed free space two-port photonic vector network analyzer with up to $2 \mathrm{THz}$ bandwidth," Opt. Express, vol. 29, no. 8, p. 12278, Apr. 2021.

[6]. W. Kruppa and K. F. Sodomsky, "An explicit solution for the scattering parameters of a linear two-port measured with an imperfect test set," IEEE Transactions on Microwave Theory and Techniques, vol. 19, no. 1. pp. 122123, 1971.

[7]. G. F. Engen and C. A. Hoer, "Thru-Reflect-Line: An Improved Technique for Calibrating the Dual Six-Port Automatic Network Analyzer," IEEE Trans. Microw. Theory Tech., vol. 27, no. 12, pp. 987-993, 1979.

[8]. C. A. Hoer and G. F. Engen, "On-Line Accuracy Assessment for the Dual Six-Port ANA: Extension to Nonmating Connectors," IEEE Trans. Instrum. Meas., vol. IM-36, no. 2, pp. 524-529, 1987. 
[9]. M. Mueh, S. Brandl, P. Hinz, C. Waldschmidt, and C. Damm, "Calibration Technique for THz Time-Domain Spectrometers Enabling Vectorial Scattering Parameter Measurements," IEEE Microw. Wirel. Components Lett., vol. 31, no. 6, pp. 805-807, Jun. 2021.

(C)2021 IEEE. Personal use of this material is permitted. Permission from IEEE must be obtained for all other uses, in any current or future media, including reprinting/republishing this material for advertising or promotional purposes, creating new collective works, for resale or redistribution to servers or lists, or reuse of any copyrighted component of this work in other works.

Published article: F. R. Faridi and S. Preu, "Characterization of a Crossed Dipole Resonator Array using a Pulsed Free Space Two-Port Photonic VNA," 2021 46th International Conference on Infrared, Millimeter and Terahertz Waves (IRMMW-THz), 2021, pp. 1-2, doi: 10.1109/IRMMW-THz50926.2021.9567495.

Available under only the rights of use according to UrhG. 